\title{
Biodistribution of rhodamine B fluorescence-labeled cationic nanoparticles in rats
}

Knudsen, Kristina Bram; Northeved, Helle; Gjetting, Torben; Permin, Anders ; Andresen, Thomas Lars; Wegener, Karen Malene; Rye Lam, Henrik ; Lykkesfeldt, Jens

Published in:

Journal of Nanoparticle Research

DOI:

10.1007/s11051-013-2221-1

Publication date:

2014

Document version

Early version, also known as pre-print

Citation for published version (APA):

Knudsen, K. B., Northeved, H., Gjetting, T., Permin, A., Andresen, T. L., Wegener, K. M., Rye Lam, H., \&

Lykkesfeldt, J. (2014). Biodistribution of rhodamine B fluorescence-labeled cationic nanoparticles in rats. Journal of Nanoparticle Research, 16(1), [2221]. https://doi.org/10.1007/s11051-013-2221-1 


\title{
Biodistribution of rhodamine B fluorescence-labeled cationic nanoparticles in rats
}

\author{
Kristina Bram Knudsen • Helle Northeved • Torben Gjetting • \\ Anders Permin • Thomas L. Andresen • Karen Malene Wegener • \\ Henrik Rye Lam · Jens Lykkesfeldt
}

Received: 23 August 2013/Accepted: 18 December 2013/Published online: 4 January 2014

(C) Springer Science+Business Media Dordrecht 2014

\begin{abstract}
We investigated the biodistribution following the administration of nanosized (about 50 and $90 \mathrm{~nm})$ cationic $(\zeta:+30$ and $+50 \mathrm{mV})$ micelles and liposomes intended for drug delivery. The particles were stable and well characterized with respect to size and $\zeta$ potential. Ten 5- to 6-week-old male rats were used. The animals were randomly allocated to five groups receiving either cationic micelles or cationic liposomes by single intravenous (IV) administration at a dose of $100 \mathrm{mg} / \mathrm{kg}$ bodyweight by single intracerebroventricular (ICV) injection at a dose of $50 \mu \mathrm{g}$ or no treatment. ICV administration was used to study local distribution in the brain and IV administration to study the systemic distribution of the particles. For both types of particles, ICV administration showed distribution in all ventricles in the brain while IV delivery displayed distribution to the major organs liver, spleen, kidney and lung, but not to the brain. Our
\end{abstract}

K. B. Knudsen · J. Lykkesfeldt ( $\square)$

Faculty of Health and Medical Sciences, Department of Veterinary Disease Biology, University of Copenhagen, Ridebanevej 9, 1, 1870 Frederiksberg C, Denmark e-mail: jopl@sund.ku.dk

K. B. Knudsen · H. Northeved - K. M. Wegener

H. Lundbeck A/S, Valby, Denmark

Present Address:

K. B. Knudsen

National Research Center for the Working Environment, Copenhagen, Denmark data suggest that cationic micelles and liposomes are widely distributed in the body, indicating that these could potentially be used as drug delivery carriers to the major organs, but they do not cross the blood-brain barrier to a significant extent, without a targeting ligand attached. However, they are able to persist in the ventricles of the brain up to $24 \mathrm{~h}$ after ICV administration, demonstrating a new ability.

Keywords Micelles - Liposomes ·

Distribution · Nanotechnology $\cdot$ Rhodamine B

\section{Introduction}

Nanoparticles (NPs) are promising candidates in drug delivery systems due to their unique physio-chemical properties, which enables them to target normally

\author{
T. Gjetting - T. L. Andresen \\ Center for Nanomedicine and Theranostics, DTU \\ Nanotech, Technical University of Denmark, Lyngby, \\ Denmark \\ A. Permin \\ DTU FOOD, Technical University of Denmark, Søborg, \\ Denmark \\ H. R. Lam \\ Environment and Toxicology, DHI, Hørsholm, Denmark
}


inaccessible areas and transport hydrophilic or lipophilic drugs or genes to these locations (Andresen et al. 2005; Donaldson 2006; Kagan et al. 2005; Kreuter and Gelperina 2008; Roney et al. 2005). There has been great interest in using NPs to target specific disease areas such as tumor tissue and areas affected by neurodegenerative disorders in the central nervous system (CNS) (Drummond et al. 1999; Re et al. 2012; Schnyder and Huwyler 2005).

Drug delivery to the brain, to treat neurological and psychiatric disorders, is complicated by the presence of the blood-brain barrier (BBB) that selectively blocks the passive transport of hydrophilic and charged compounds (Lo et al. 2001). Several actions are being taken in constructing particles which could penetrate the BBB without disruption, thereby obtaining a desired therapeutic effect in the brain tissue (Costantino et al. 2005; Pardridge 2012; Tosi et al. 2007; Tosi et al. 2008). Ideally, the particles should not contribute to therapy or toxicity by themselves, but rather deliver the therapeutic agent and subsequently be eliminated by phagocytic cells. However, the control of such delivery and the subsequent distribution of NPs in the brain are at present poorly understood.

Not all NPs are potential candidates for drug delivery. The NPs that show great potential as drug carriers include lipid-like particles such as liposomes, which are already used as drug delivery systems in treatment of cancer (Andresen et al. 2010), but also polymeric micelles are promising systems for different drug delivery applications (Park et al. 2008). Knowledge of biodistribution is an integral part of the evaluation of new drug candidates to assess if the right organs/tissues are targeted and to investigate potential organ toxicity (Oberdorster et al. 2005). Distribution can be altered by, e.g., binding of proteins in the blood or cerebrospinal fluid (CSF), which can change the size or other properties of the particles and thereby the target tissue concentration (Moghimi and Hunter 2001; Oberdorster et al. 2005; Owens and Peppas 2006).

The relationship between particle size and distribution of gold particles has previously been evaluated. Larger particles around $100 \mathrm{~nm}$ were distributed to major organs such as liver, spleen and kidney, after IV injection, whereas smaller particles around $50 \mathrm{~nm}$ were also distributed to the lung and heart. Ten $\mathrm{nm}$ particles were shown to be widespread in the tissues after $24 \mathrm{~h}$; here De Jong et al. (2008) detected gold particles in all organs evaluated after IV injection including the brain (de Jong et al. 2008). Silver NPs with a size of $14 \mathrm{~nm}$ were after oral administration found in major organs such as liver, kidneys, lung and even found in brain (Loeschner et al. 2011).

PEGylation (coating with polyethylene glycol) of particles has been known to prolong the circulation time by shielding the particles from being cleared rapidly by the reticular system (RES), but this coating has also been reported to alter the biodistribution of the particles (Moghimi and Szebeni 2003; Niidome et al. 2006; Owens and Peppas 2006). Other factors than size and coating influencing the distribution includes the surface charge. For example, cationic effects have been suggested to trap nanosized metal dendrimers in the kidneys up to 4 days (Balogh et al. 2007). Cationic effects are, e.g., desirable as an effective binding mechanism to negatively charged DNA (Dai et al. 2011; Gjetting et al. 2011), but cationic effects have also shown to facilitate cellular uptake (Thurston et al. 1998).

We recently reported toxicity of cationic NPs in the hippocampus of the rat following intracerebroventricular (ICV) administration (Knudsen et al. 2014). In the present study, we have used NPs fluorescence labeled with rhodamine B to investigate the systemic and CNS distribution of cationic micelles and liposomes following IV or ICV injection, respectively, since to our knowledge, there is limited information in the literature on the distribution of micelles and liposomes in vivo, in particular to the brain.

\section{Materials and methods}

Nanoparticles

\section{Preparation of rhodamine B-labeled cationic liposomes}

Lipids were purchased from Avanti Polar Lipids (Alabaster, AL, USA). Cationic liposomes were prepared from $N$-[1-(2,3-dioleyl)propyl]- $N, N, N$-trimethylammonium chloride (DOTAP, synthetic, $50 \%$ $\mathrm{w} / \mathrm{w}$ ) and cholesterol (plant source, $49.75 \% \mathrm{w} / \mathrm{w}$ ) and 1,2-dioleoyl-sn-glycero-3-phosphoethanolamine- $N$ (lissamine rhodamine B sulfonyl) (ammonium salt, DOPE-RhB, synthetic, $0.25 \% \mathrm{~W} / \mathrm{W}$ ) at a concentration of $10 \mathrm{mg} / \mathrm{ml}$ in sterile phosphate-buffered saline (PBS) as previously described (Gjetting et al. 2010). Briefly, lipids were dissolved in chloroform and mixed 
in a glass vial. The solvent was evaporated under an argon gas stream and the lipid film was dried in highvacuum overnight. Hydration was performed in the PBS at $50{ }^{\circ} \mathrm{C}$ for $2 \mathrm{~h}$ followed by sonication for $30 \mathrm{~min}$ using a Bransonic water bath (MT-1510, $42 \mathrm{kHz}$, $80 \mathrm{~W}$, setting “sonics", Branson Ultrasonics, Danbury, CT, USA).

For characterization, $20 \mu \mathrm{l}$ of cationic liposomes were diluted in 1.8-ml buffer (5\% glucose containing $10 \mathrm{mM}$ Na-HEPES, pH 7.4) in disposable cuvettes (Sarstedt, Nümbrecth, Germany) and the size distribution of the NPs was measured by dynamic light scattering (DLS) using a ZetaPALS (Zeta Potential Analyzer) (Brookhaven Instruments, NY, USA) at standard settings, typically 10 sub-runs of $30 \mathrm{~s}$. Data were fitted using the built-in software to estimate size and polydispersity index (PDI). Subsequently, $\zeta$ potential was measured with the same sample using a conditioned electrode, typically by 10 runs, observing the relative residual from model fitting, $p<0.04$. A typical preparation had an average size of $90 \pm 1 \mathrm{~nm}$ (mean \pm SEM) and a $\zeta$ potential of $+50 \pm 2 \mathrm{mV}$. A polydispersity index of 0.1 was measured for the sample, which indicated that the cationic liposome preparation was highly monodisperse. CryoTEM images of DOTAP/cholesterol liposomes have been published previously (Templeton et al. 1997). A structured figure illustrates the construction of the liposome in Fig. 1a.

\section{Preparation of cationic micelles}

The amphiphilic triblock-copolymer poly(methyl methacrylate)-b-poly(aminoethyl methacrylate)-b- poly(ethylene
Fig. 1 a Cationic liposomes: chemical structures and molar composition: DOTAP (50\%), cholesterol (49.75\%) and DOPE-RhB $(0.25 \%)$. b Cationic micelles: structures and reaction scheme of synthesized triblockcopolymer PMMA-bPAEMA-b-PEG with fluorophore RhB-ITCapproximately $0.25 \%$ of polymers are modified. Upon hydration, both materials self-organize into fluorescent nanoparticles

\section{a}

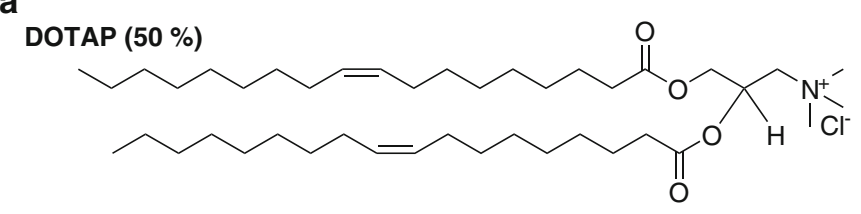

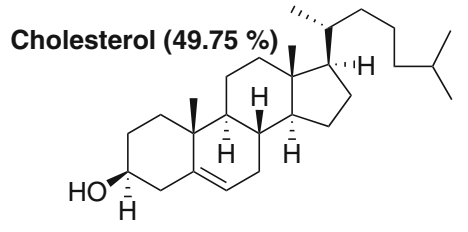

DOPE-RhB (0.25 \%)

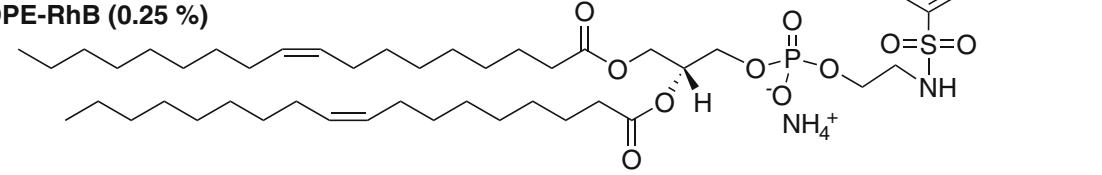

b PMMA-b-PAEMA-b-PEG (99.75 \%)

PMMA-b-PAEMA(RhB)-b-PEG (0.25\%)

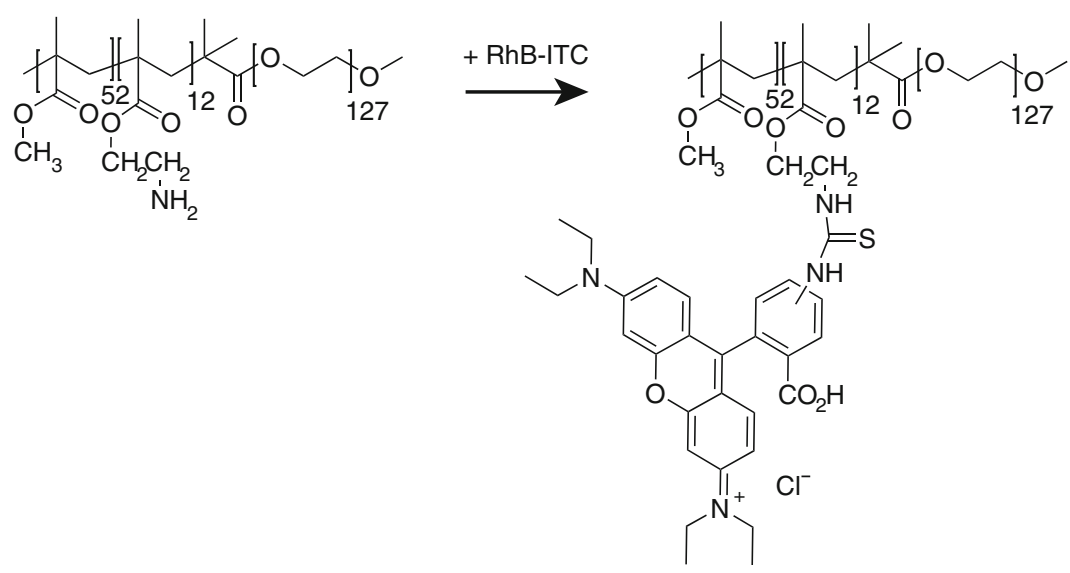


glycol) (PMMA-b-PAEMA-b-PEG) $(500 \mathrm{mg}, 40 \mu \mathrm{mol})$ synthesized by our previously described method (Kumar et al. 2012; Kumar et al. 2013), was dissolved in $14 \mathrm{ml}$ of dimethylformamide. To this clear polymer solution, $4 \mathrm{ml}$ of MilliQ water was added under stirring within a time interval of $30 \mathrm{~min}$ followed by dropwise addition of another $24 \mathrm{ml}$ MilliQ water to facilitate homogenous micelle formation. The cloudy micelle dispersion was then transferred into a dialysis tube $(\mathrm{MWCO}=12 \mathrm{kDa})$ and dialysis was performed against MilliQ water for 2 days [size: $51 \pm 2 \mathrm{~nm}$, PDI 0.09 , $\zeta$ potential: $32 \pm 2 \mathrm{mV}$, as measured in glucose (5\%), Na-HEPES (10 mM, pH 7.4)] and then against PBS buffer (pH 7.4) for another 3 days. The final micelle concentration was $9.9 \mathrm{mg} / \mathrm{ml}$ (measured as dry mass after the dialysis against water). The critical micelle concentration has previously been determined to be $<10 \mathrm{mg} / \mathrm{l}$ for this class of triblock copolymer, and the structural properties of the triblock copolymer have been evaluated by cryoTEM, which showed the spherical morphology for the micelles (Kumar et al. 2012).

\section{Preparation of rhodamine B-labeled micelles}

Ten milliliters of PMMA-b-PAEMA-b-PEG micelle preparation $(9.9 \mathrm{mg} / \mathrm{ml})$ from above were dialyzed against sodium carbonate buffer $(50 \mathrm{mM}, \mathrm{pH}$ 9) for 2 days. The micelle dispersion was transferred into a round bottom flask and rhodamine $\mathrm{B}$ isothiocyanate [Sigma, $0.2 \mu \mathrm{mol}$ from a $1 \mathrm{mg} / \mathrm{ml}$ stock solution in dimethyl sulfoxide (DMSO)] was added and stirred at room temperature for $12 \mathrm{~h}$ (Kumar et al. 2013). The micelle preparation was transferred to a dialysis tube with a molecular weight cut-off at $12 \mathrm{kDa}$ and dialysis was performed against the carbonate buffer for 3 days to remove unreacted fluorophore. Subsequently, dialysis against MilliQ water for another 3 days was followed by quantification and characterization [size: $51 \pm 2 \mathrm{~nm}$, PDI: 0.09 , $\zeta$ potential: $30 \pm 2 \mathrm{mV}$, measured in glucose $(5 \%)$, Na-HEPES $(10 \mathrm{mM}, \mathrm{pH}$ 7.4)] and finally against PBS buffer ( $\mathrm{pH}$ 7.4) for another 3 days. The final micelle concentration was $9.8 \mathrm{mg} / \mathrm{ml}$. The cationic micelles dispersed in the PBS buffer were transferred into a sterile tube. The coupling efficiency of the conjugation was estimated to be $50 \%$ resulting in a micelle preparation where $1 / 400(0.25 \%)$ of the monomeric triblock-copolymer units in the micelle contains rhodamine B. A structured figure illustrates the construction of the micelle in Fig. 1b.

The stability of the particles was tested after storage for 1 month without any change in size or $\zeta$ potential (data not shown). The formulations were therefore considered stable for at least the duration of the study.

\section{Animals}

Ten male Han Wistar rats (Crl:WI (Han)) were obtained from Charles River (Wiga GmbH, Germany). The rats were 4 weeks old at arrival and were housed in humidity and temperature-controlled ventilated cupboards (Scantainers, Scanbur technology, Karlslunde, Denmark) with two rats per cage. They were acclimatized for 2 weeks prior to examination and a 12-h day/night cycle was maintained. A standard rodent diet (Altromin 1324, Brogaarden, Denmark) and water were provided ad libitum. Following acclimatization, the animals were randomly divided into five groups $(n=2)$ receiving either cationic micelles or cationic liposomes by single IV administration at a dose of $100 \mathrm{mg} / \mathrm{kg}$ bodyweight, the same particles by single ICV injection at a dose of $50 \mu \mathrm{g}$, or no treatment. Body weights were recorded three times (every second day) in the week before treatment and on a daily basis during the study. Food consumption was recorded per cage on a weekly basis. Cage-side clinical observations were conducted several times daily during the dosing period and changes were recorded.

All experiments were conducted according to the Danish Federal guidelines for use and care of laboratory animals and were approved by the Danish Executive Order No. 1306 of 23 November 2007 on Animal Testing.

\section{Administration}

The dosages were determined on preliminary studies and were considered to be tolerable during short term administration in rats. For the ICV administration, animals were anesthetized with a 3:1 mixture of HypNorm and Dormicum (Roche, Basel, Schweiz) at a dose volume of $2.5 \mathrm{ml} / \mathrm{kg}$ bodyweight subcutaneously. Subsequently, using a stereotactic frame, a hole was drilled in the skull on top of the right lateral ventricle, at the following coordinates measured from Bregma: anterior/posterior: $-0.8 \mathrm{~mm}$; medial/lateral: 
$-1.4 \mathrm{~mm}$; dorsal: $-3.6 \mathrm{~mm}$ (Paxinos and Watson 2008). A $10-\mu 1$ Hamilton syringe gauge $26 \mathrm{~S}$ was connected to the device and a volume of $5 \mu \mathrm{l}$ of the NP formulation $(\sim 10 \mathrm{mg} / \mathrm{ml})$ was injected into the lateral ventricle at a rate of $2 \mu \mathrm{l} / \mathrm{min}$ corresponding to a dose of NPs of $50 \mu \mathrm{g}$. For the IV administration, the NPs formulation $(\sim 10 \mathrm{mg} / \mathrm{ml})$ was given once in a volume of $10 \mathrm{ml} / \mathrm{kg}$ bodyweight corresponding to a NP dose of $100 \mathrm{mg} / \mathrm{kg}$ bodyweight. All particles were diluted in PBS. Control animals were non-treated and functioned as a control with respect to background fluorescence.

Sample collection

Twenty-four hours after injection, animals were anesthetized with Avertin (Ampliqon, Odense, Denmark) using a dose of $10 \mathrm{ml} / \mathrm{kg}$ bodyweight intraperitoneally. Animals were sacrificed by exsanguination while still anesthetized. Organs (liver, lung, spleen, kidneys and brain) were isolated and examined macroscopically.

\section{Histology and biodistribution}

Organs (brain, liver, lung, spleen and kidneys) were frozen solid on crushed dry ice, to avoid crystallization in the tissue, and then packaged in gold bags and stored at $-80{ }^{\circ} \mathrm{C}$. Frozen tissue was moved to a $-20{ }^{\circ} \mathrm{C}$ freezer, $24 \mathrm{~h}$ before sectioning. Organs were embedded in Tissue Tec (Sukura, AJ Alphen aan den Rijn, The Netherlands) and sections were cut at $10 \mu \mathrm{m}$ on a Microtome (Leica Microsystems, Wetzlar, Germany). The tissue sections were examined with a Nikon Eclipse E1000 fluorescence microscope (Nikon Instruments Europe B.V., Surrey, England) using Cyanine 3 (CY3) filter excitation wavelength $550 \mathrm{~nm}$ and emission at $570 \mathrm{~nm}$. Microphotographs were taken with a ProRes c14 Jenoptik camera (Jenoptik Optical Systems, Jena, Germany) using the image program ImagePRO 6.2 (Media Cybernetics Inc., Bethesda, MD, USA). The fluorescence was evaluated qualitatively.

\section{Results}

Control animals were used to evaluate potential background fluorescence to correct for this, when analyzing the different treatment groups (Fig. 2). IV administration was used to test systemic biodistribution of the NPs. Following IV administration, fluorescent particles could be detected in all animals $24 \mathrm{~h}$ after administration in the collected organs.

In animals given rhodamine B-labeled micelles IV, a high content of particles was observed in the liver (Fig. 3a). The particles were distributed throughout the liver and were located in portal areas and interlobular tissue. The particles appeared to be present in the vessels, as well as in the cytoplasm of hepatocytes. In the spleen, the content appeared to be as high as in the liver and the particles were primarily located in the red pulp (Fig. 3b). In the lung tissue, the micelles were located in high numbers in a few areas (Fig. 3c). In the kidneys, a lower content of particles was observed when compared to the liver and spleen. The particles were mainly located in the glomeruli (Fig. 3d). In general, the micelles were widely distributed in compartments of the major organs after IV administration (Fig. 3a-d).

In the brain, a few micelles were observed in the choroid plexus of the lateral ventricle and in the meninges (Fig. 3e, f). A few particles were also located in the third ventricle and in the cortex of the brain (Fig. 3g, h).

Following IV injection with rhodamine B-labeled liposomes, a high number of particles were observed throughout the liver (Fig. 4a). These particles appeared to be located in the portal areas. In the spleen, the content was similar to that found in the liver, and the clusters of liposomes were located primarily in the red pulp (Fig. 4b). In the lung, particles were seen in a few smaller areas (Fig. 4c), while the kidney showed particles located in the glomeruli (Fig. 4d). In general, the liposomes were not as widely distributed as the micelles after IV administration, the liposomes were more packed in clusters (Fig. 4a-d) than observed with the micelles.

In the brain, the liposomes were detected in the choroid plexus of the lateral ventricles (Fig. 4e). Particles were also found in the meninges (Fig. 4f, g). Finally, a few particles were located in the cortex (Fig. 4h).

In animals injected ICV with micelles, a few particles were detected in the liver and in the kidney (images not shown). Particles may have been present in the spleen and lung, but these were not positively identified during microscopy. In the brain, a high content of micelles were found in the ventricular system of the brain. They were detected rostrally as well as caudally to the injection site 


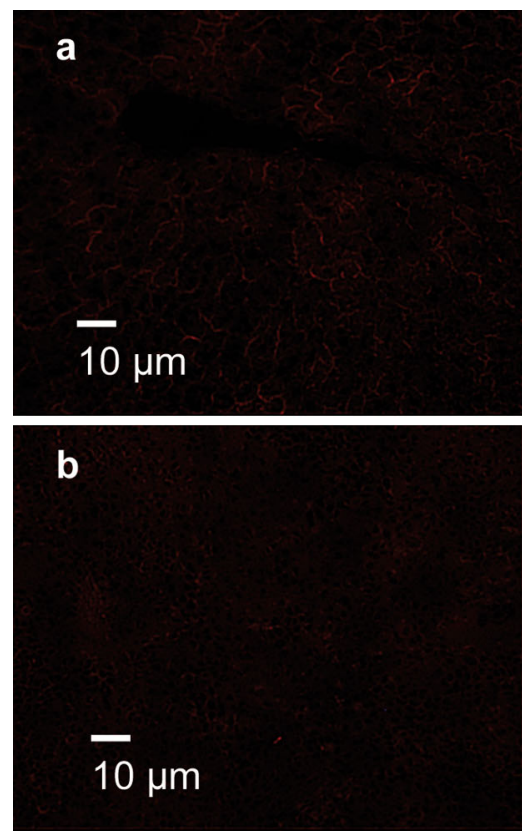

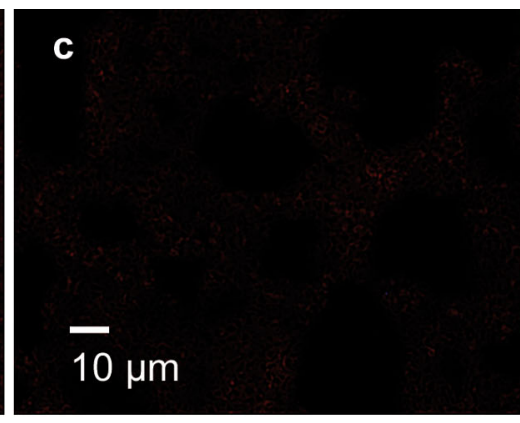

d

$10 \mu \mathrm{m}$

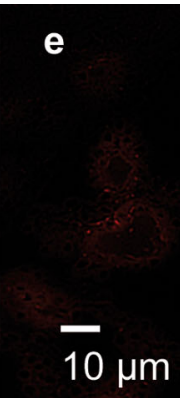

$\mathbf{f}$
Fig. 2 Organ sections from non-treated control animals seen through CY3 filter for fluorescence excitation at $550 \mathrm{~nm}$; emission $570 \mathrm{~nm}$. The micrographs illustrate the background fluorescence. Objectives $\times 20$. a Liver around the portal area, b spleen, c lung, $\mathbf{d}$ kidney cortex with glomeruli, e the lateral ventricle in the brain with choroid plexus and $\mathbf{f}$ brain meninges
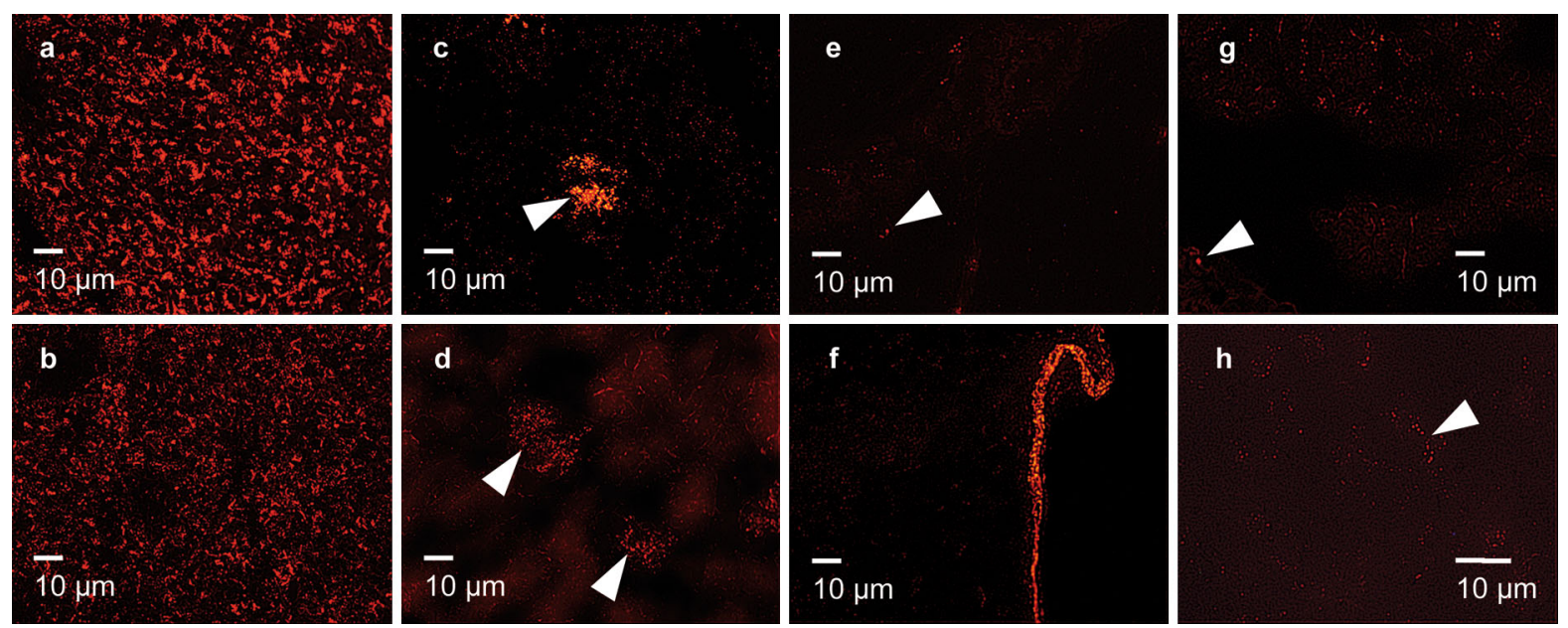

Fig. 3 Organ sections obtained 24-h post IV administration of rhodamine B-marked micelles seen through a CY3 filter for fluorescence (excitation $550 \mathrm{~nm}$; emission $570 \mathrm{~nm}$ ). a-g Objectives $\times 20$, $\mathbf{h}$ objective $\times 40$. a Liver, fluorescent particles in the portal areas, b spleen, fluorescent particles in the red pulp, c lung, area with clusters of fluorescent particles, d kidney,

and were located in both lateral ventricles, the third ventricle, the cerebral aqueduct and in the fourth ventricle in cerebellum (Fig. 5a, b).

After ICV administration of liposomes, a few particle clusters were found in the lung tissue. In

fluorescent particles in the glomeruli, e Lateral ventricle in the brain. Choroid plexus with a few fluorescent particles and f brain, fluorescent particles in the meninges. $\mathrm{g}$ Few particles located in the third ventricle of the brain. $\mathbf{h}$ Few particles located in the cortex of the brain

addition, a few particles were observed in the portal area of the liver, in the kidney and in the spleen (images not shown). In the brain, a high content of liposomes was observed in the ventricular system and distributed in a similar manner as the micelles (Fig. 5c). 

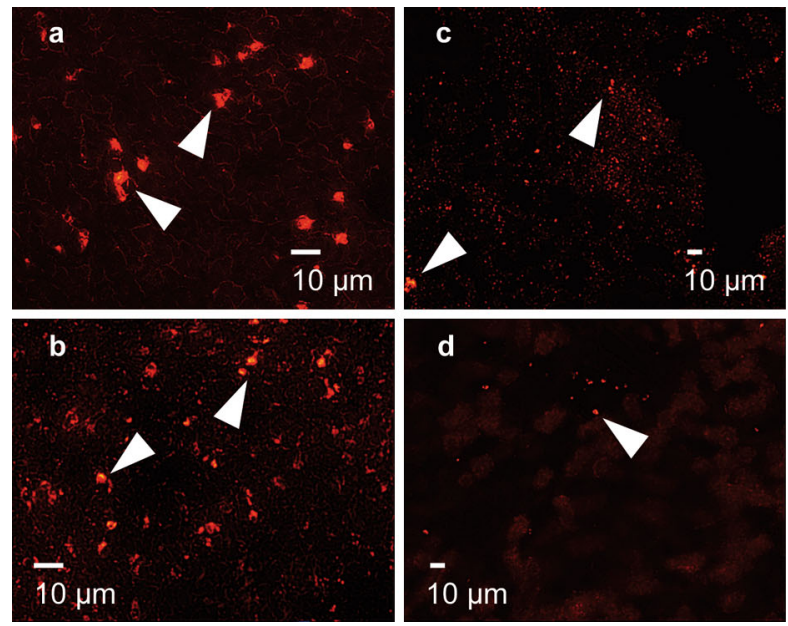

Fig. 4 Organ sections obtained 24-h post IV administration of rhodamine B-marked liposomes seen through a CY3 filter for fluorescence (excitation $550 \mathrm{~nm}$; emission $570 \mathrm{~nm}$ ). a, b, e, g, h Objectives $\times 20$; $\mathbf{c}, \mathbf{d}$ objective $\times 10$; $\mathbf{f}$ objective $\times 40$. a Liver, portal area with clusters of fluorescent particles, b spleen, clusters of fluorescent particles in the red pulp, $\mathbf{c}$ lung, small area with cluster of fluorescent particles, d kidney, fluorescent
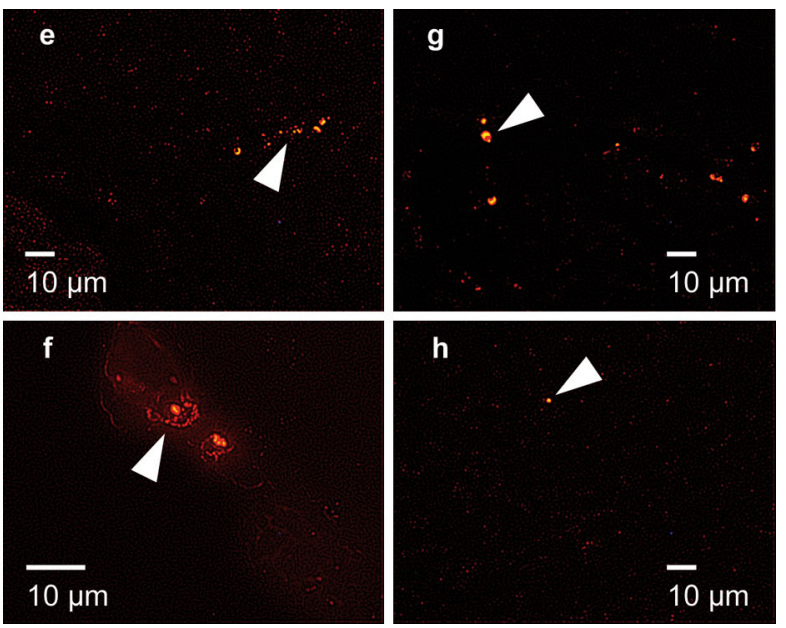

particles in the glomeruli, e lateral ventricle in the brain. Choroid plexus with a few fluorescent particles. f Brain, fluorescent particles in the meninges, $\mathbf{g}$ fluorescent particles located in the leptomeningeal space between the lateral and the third ventricle, and $\mathbf{h}$ single fluorescent particles located in the cortex
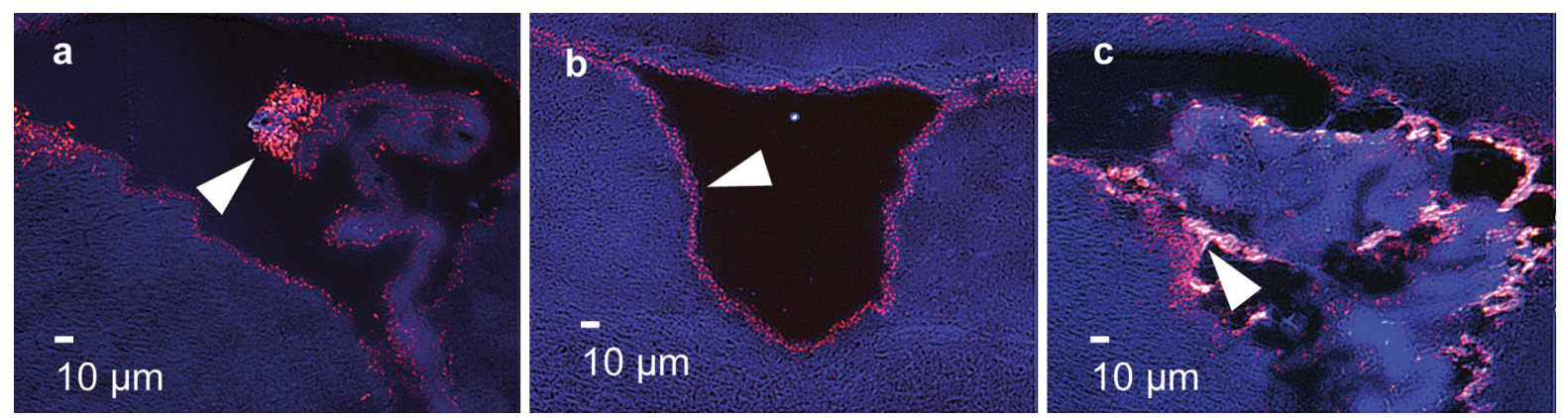

Fig. 5 Brain sections obtained 24-h post ICV administration of rhodamine B-marked micelles or liposomes seen through a CY3 filter for fluorescence (excitation $550 \mathrm{~nm}$; emission $570 \mathrm{~nm}$ ). Objectives $\times 10$. a Lateral ventricles of the brain at the injection site. Fluorescent micelles are located at the choroid plexus and

The micelles and liposomes found in the brain ventricles were located both at the choroid plexus and along the walls of the brain ventricles (Fig. 5a-c).

Findings are summed up in Table 1.

\section{Discussion}

The present study was designed to mimic BBB transport by injecting particles directly into the brain by ICV, thereby surpassing the BBB and to compare along the ventricle wall, $\mathbf{b}$ fourth brain ventricle with fluorescent micelles along the ventricle wall and $\mathbf{c}$ lateral ventricles of the brain at the injection site. Fluorescent liposomes are located along the ventricle wall

this with the biodistribution after IV administration. Twenty-four hours after administration, the particles were located in major organs after IV administration and in the ventricles of the brain after ICV administration. The protection by PEGylation offers the particles a half-life up to $24 \mathrm{~h}$ (Moghimi et al. 2001). The study duration of $24 \mathrm{~h}$ was chosen to balance the ability of the particles to reach the target sites and their half-life. Furthermore, the NP dose of $100 \mathrm{mg} / \mathrm{kg}$ bodyweight should facilitate the micellar structures that would not dissociate due to dilution given a 
Table 1 Tissue distribution of particles

\begin{tabular}{|c|c|c|c|c|c|}
\hline Tissue/no. of animals affected & $\begin{array}{l}\text { Control } \\
n=2\end{array}$ & $\begin{array}{l}\text { ICV cationic } \\
\text { micelle } \\
n=2\end{array}$ & $\begin{array}{l}\text { IV cationic } \\
\text { micelle } \\
n=2\end{array}$ & $\begin{array}{l}\text { ICV cationic } \\
\text { liposome } \\
n=2\end{array}$ & $\begin{array}{l}\text { IV cationic } \\
\text { liposome } \\
n=2\end{array}$ \\
\hline \multicolumn{6}{|l|}{ Liver } \\
\hline $\begin{array}{l}\text { Fluorescent particles located in portal veins or } \\
\text { interlobular connective tissue }\end{array}$ & 0 & $2^{\mathrm{a}}$ & 2 & $2^{\mathrm{a}}$ & 2 \\
\hline \multicolumn{6}{|l|}{ Spleen } \\
\hline Fluorescent particles located primarily in the red pulp. & 0 & 0 & 2 & $2^{\mathrm{a}}$ & 2 \\
\hline \multicolumn{6}{|l|}{ Kidney } \\
\hline $\begin{array}{l}\text { Fluorescent particles located in and around the } \\
\text { glomerulus }\end{array}$ & 0 & $2^{\mathrm{a}}$ & 2 & $2^{\mathrm{a}}$ & 2 \\
\hline \multicolumn{6}{|l|}{ Lung } \\
\hline Fluorescent particles located in blood vessels & 0 & 0 & 2 & $2^{\mathrm{a}}$ & 2 \\
\hline \multicolumn{6}{|l|}{ Brain } \\
\hline Fluorescent particles located in all ventricles & 0 & 2 & $2^{\mathrm{b}}$ & 2 & $2^{\mathrm{b}}$ \\
\hline
\end{tabular}

putative critical micelle concentration around $10 \mathrm{mg} / \mathrm{l}$. Rhodamine B applied as a fluorophore had been successfully used in other studies testing the distribution of NPs (Harrison et al. 2012; Krasnici et al. 2003; Vergoni et al. 2009). Toxicity of the NPs per se was not assessed in this study as the fluorophore alone could have an independent toxic effect (Avanti Polar Lipids Inc. 2013). The fluorophore was built into the lipid membranes of the NPs, therefore the RhB does not drop off the NPs during the transport thereby linking the observed fluorescent signals to the presence of the NPs themselves. The fluorescent NPs were still visible $24 \mathrm{~h}$ after administration. This could indicate that the particles are still circulating, but they could potentially also be visible after being engulfed by cells.

The development of safe drug delivery systems to both CNS and systemic targets is a high priority in modern drug development, but also the specific delivery of agents to ensure an effective and long lasting pharmacological effect. Biodistribution studies are a way to determine target areas for potential drug carriers and may also provide an information on organ accumulation, clearance and safe use. Serum proteins might alter the particle size, surface charge, and other properties, that may change the particle distribution and pharmacokinetics. Thus, cationic liposomes have been shown to interact with anionic components in the blood plasma thereby accelerating their clearance rate and lowering their circulation time. In contrast, PEGylation has been shown to minimize such protein interactions and prolong the half-life of the NP species (Zhao et al. 2011).

In this study, PEGylated micelles and non-PEGylated liposomes were used. A similar distribution pattern was observed $24 \mathrm{~h}$ post administration. Both types of NPs were found in liver, lung, spleen, and kidney and to a lesser degree in meninges in the brain after the IV injections and primarily in the brain ventricles after ICV injections. Compared to a study by de Jong et al. (2008), who did not detect gold particles at the size of $100 \mathrm{~nm}$ in lung tissue after IV injection, we detected particles with a size around 90 and $50 \mathrm{~nm}$ in areas of the lung. Theoretically, nonPEGylated liposomes should be cleared rapidly from the blood after IV injection by elements of the RES (Moghimi and Szebeni 2003). However, the relatively high concentrations used may have increased their half-life as some dose dependency has previously been observed for similar NPs. Thus, Hong et al. (1999) compared PEGylated and non-PEGylated liposomal Doxorubicin and found that the half-life increased from $12.5 \mathrm{~h}$ using a dose of $6 \mathrm{mg} / \mathrm{kg}$ up to $31.7 \mathrm{~h}$ when a dose of $10 \mathrm{mg} / \mathrm{kg}$ bodyweight was used.

The cationic properties may increase cellular uptake in general as seen by Zhao et al. (2011), who found that cationic liposomes $(85-140 \mathrm{~nm}$ ) were effectively taken up by rat aortic endothelial cells, 
possibly by endocytosis involving electrostatic interactions between cells and liposomes (Zhao et al. 2011). A similar mechanism could explain the apparent uptake by liver hepatocytes in the present study. However, in contrast to our observations, Zhao et al. found that cationic liposomes were preferentially distributed to the lungs when compared with liver, spleen and kidney following IV injections. This was believed to be caused by the cationic surface, which may interact with anionic substances in the serum, and thereby causing the NPs to form aggregates, which could accumulate in the lungs (Zhao et al. 2011). A different distribution pattern was observed in the present study where the distribution favored the liver and spleen. However, Zhao et al. (2011) observed better distribution, when particles were protected by PEGylation, as observed with the PEGylated micelles in the present study. Here, the PEGylation also protected the particles from aggregation. The observations in the present study confirm that PEGylation protects from aggregation compared to observations with the liposomes, where clusters were more observed compared with micelles. When the NPs start to form aggregates, their size increase and this could increase the clearance rate by uptake by RES, and cleared to liver and spleen as observed (Lian and Ho 2001).

Balogh found that cationic gold particles $(5 \mathrm{~nm})$ accumulate more in the kidney, while anionic accumulate more in liver. They suggest that the cationic surface charge decreases targeting to the liver (Balogh et al. 2007). In this study, a more pronounced accumulation was found in liver than in kidney with cationic particles. It is not easy to compare different particles, but this difference in accumulation could be due to the time point investigated, when observing clearance rate or difference in NP characteristics, such as size, agglomeration of particles, or binding of surface proteins (Hirn et al. 2011). The particles in the present study were larger, and this could influence their distribution, since smaller NPs may avoid immune recognition (Balogh et al. 2007), thereby having an altered distribution pattern.

In the current study, the NPs are transported via the blood by systemic circulation to major organs such as the liver and spleen, where the highest fluorescence signals were observed. Here, the NPs may have been trapped. For future studies, collection of feces and urine as well as looking at other organs or tissues could provide more detailed information about the distribution and degradation of these NPs.

Drug transport from blood to the CSF is regulated by the choroid plexus, while transport from blood to the brain interstitium is regulated by the BBB (Pardridge 2011). In the rat, the CSF volume is around $90 \mu \mathrm{l}$ (Pardridge 2011) and this volume is renewed about 11 times daily (Johanson et al. 2008). We found particles present in the brain ventricles $24 \mathrm{~h}$ after ICV administration. This indicates that the particles may be bound to structures in the ventricles, as they are not cleared with the same rate as CSF. After IV injection, we observed particles in the choroid plexus. The choroid plexus is more easily penetrated than the $\mathrm{BBB}$, but it cannot be concluded that these empty particles without a ligand attached crossed either the choroid plexus or the BBB and entered the interstitial fluid (Pardridge 2011). Sarin et al. (2008) has proposed that particles larger than $\sim 12 \mathrm{~nm}$ are not able to transverse the BBB (Sarin et al. 2008), which could explain why noteworthy fluorescence in the brain was not detected in the present study following IV administration, and this indicates that the construct would require a targeting ligand in future experiments, if the size cannot be reduced. However, as micellar/ lipid structures are dynamic, deformable and may lose their cargo during trafficking in the body, e.g., when they are squeezing through cellular barriers, it cannot be ruled out that the fluorescent tracer was lost during crossing of the $\mathrm{BBB}$, although the construct of the tracer was designed not to come off the NPs as secured by a lipid bond to $\mathrm{RhB}$ tracer.

A few particles were detected in liver after ICV injection, which could indicate a clearance from the brain ventricles. However, it may also be that particles were mistakenly injected into blood vessel during the administration.

In one of our recent studies, administration of cationic particles was found to be associated with toxicity in terms of cell death and inflammation in the hippocampal area when injected ICV. However, no toxicity was detected in other major organs examined (liver, kidney, lung and spleen) after IV administration (Knudsen et al. 2014). This study shows that the cationic NPs are distributed well in major organ systems and further supports that the NPs could eventually be used for systemic drug delivery. Furthermore, that there are correlation between the persistence of the particles (at least for $24 \mathrm{~h}$ ) and the 
toxicity in the brain observed in earlier investigations (Knudsen et al. 2014). However, the present study does not allow conclusions on potential tissue accumulation over time and it should also be acknowledged that different time points and doses may give rise to different distribution patterns.

In conclusion, this study has shown a distribution of RhB-labeled lipid NPs to major organs (liver, spleen, kidneys and lung) after IV injection suggesting that such particles could potentially be used as drug delivery carriers to target these sites. The study has also indicated that if particles are constructed to cross $\mathrm{BBB}$, lipid-based particles could persist in the ventricles and potentially bind to specific elements in the tissue or the CSF.

Acknowledgments The excellent assistance of the Animal Facility, Section of Neurobiology and Section of Pathology and Clinical Pathology at H. Lundbeck A/S, Pramod Kumar EK and Jonas Rosager Henriksen from DTU Nanotech and the contribution from DHI are gratefully acknowledged. This work was supported by H. Lundbeck A/S, DHI and Faculty of Health and Medical Sciences, University of Copenhagen.

Conflict of interest The authors declare no conflicts of interest that could influence the present study.

\section{References}

Andresen TL, Jensen SS, Jorgensen K (2005) Advanced strategies in liposomal cancer therapy: problems and prospects of active and tumor specific drug release. Prog Lipid Res 44:168-197

Andresen TL, Thompson DH, Kaasgaard T (2010) Enzymetriggered nanomedicine: drug release strategies in cancer therapy. Mol Membr Biol 27:7353-7363

Avanti Polar Lipids Inc. (2013) MSDS RhB. http://avantilipids. com/MSDS/msds.php?ProdNum=810150P\&format=raw

Balogh L, Nigavekar SS, Nair BM, Lesniak W, Zhang C, Sung LY, Kariapper MS, El-Jawahri A, Llanes M, Bolton B, Mamou F, Tan W, Hutson A, Minc L, Khan MK (2007) Significant effect of size on the in vivo biodistribution of gold composite nanodevices in mouse tumor models. Nanomedicine 3:4281-4296

Costantino L, Gandolfi F, Tosi G, Rivasi F, Vandelli MA, Forni F (2005) Peptide-derivatized biodegradable nanoparticles able to cross the blood-brain barrier. J Control Rel 108:184-196

Dai Z, Gjetting T, Mattebjerg MA, Wu C, Andresen TL (2011) Elucidating the interplay between DNA-condensing and free polycations in gene transfection through a mechanistic study of linear and branched PEI. Biomaterials 32:338626-338634

de Jong WH, Hagens WI, Krystek P, Burger MC, Sips AJ, Geertsma RE (2008) Particle size-dependent organ distribution of gold nanoparticles after intravenous administration. Biomaterials 29:121912-121919

Donaldson K (2006) Resolving the nanoparticles paradox. Nanomedicine (Lond) 1:2229-2234

Drummond DC, Meyer O, Hong K, Kirpotin DB, Papahadjopoulos D (1999) Optimizing liposomes for delivery of chemotherapeutic agents to solid tumors. Pharmacol Rev 51:4691-4743

Gjetting T, Arildsen NS, Christensen CL, Poulsen TT, Roth JA, Handlos VN, Poulsen HS (2010) In vitro and in vivo effects of polyethylene glycol (PEG)-modified lipid in DOTAP/ cholesterol-mediated gene transfection. Int $\mathrm{J}$ Nanomed 5:371-383

Gjetting T, Andresen TL, Christensen CL, Cramer F, Poulsen TT, Poulsen HS (2011) A simple protocol for preparation of a liposomal vesicle with encapsulated plasmid DNA that mediate high accumulation and reporter gene activity in tumor tissue. Results Pharma Sci 1:149-156

Harrison J, Bartlett CA, Cowin G, Nicholls PK, Evans CW, Clemons TD, Zdyrko B, Luzinov IA, Harvey AR, Iyer KS, Dunlop SA, Fitzgerald M (2012) In vivo imaging and biodistribution of multimodal polymeric nanoparticles delivered to the optic nerve. Small 8:101579-101589

Hirn S, Semmler-Behnke M, Schleh C, Wenk A, Lipka J, Schaffler M, Takenaka S, Moller W, Schmid G, Simon U, Kreyling WG (2011) Particle size-dependent and surface charge-dependent biodistribution of gold nanoparticles after intravenous administration. Eur J Pharm Biopharm 77:3407-3416

Hong RL, Huang CJ, Tseng YL, Pang VF, Chen ST, Liu JJ, Chang FH (1999) Direct comparison of liposomal doxorubicin with or without polyethylene glycol coating in C-26 tumor-bearing mice: is surface coating with polyethylene glycol beneficial? Clin Cancer Res 5:113645-113652

Johanson CE, Duncan JA III, Klinge PM, Brinker T, Stopa EG, Silverberg GD (2008) Multiplicity of cerebrospinal fluid functions: new challenges in health and disease. Cerebrospinal Fluid Res 5(10):441-450

Kagan VE, Bayir H, Shvedova AA (2005) Nanomedicine and nanotoxicology: two sides of the same coin. Nanomedicine 1:4313-4316

Knudsen KB, Northeved H, Ek PK, Permin A, Andresen TL, Larsen S, Wegener KM, Lam HR, Lykkesfeldt J (2014) Differential toxicological response to positively and negatively charged nanoparticles in the rat brain. Nanotoxicology 8:764-774. doi:10.3109/17435390.2013.829589

Krasnici S, Werner A, Eichhorn ME, Schmitt-Sody M, Pahernik SA, Sauer B, Schulze B, Teifel M, Michaelis U, Naujoks K, Dellian M (2003) Effect of the surface charge of liposomes on their uptake by angiogenic tumor vessels. Int J Cancer 105:4561-4567

Kreuter J, Gelperina S (2008) Use of nanoparticles for cerebral cancer. Tumori 94:2271-2277

Kumar EK, Almdal K, Andresen TL (2012) Synthesis and characterization of ratiometric nanosensors for $\mathrm{pH}$ quantification: a mixed micelle approach. Chem Commun (Camb) 48:394776-394778

Kumar EK, Feldborg LN, Almdal K, Andresen TL (2013) Synthesis and characterization of a micelle-based $\mathrm{pH}$ nanosensor with an unprecedented broad measurement range. Chem Mater 25:91496-91501 
Lian TF, Ho RJ (2001) Trends and developments in liposome drug delivery systems. J Pharm Sci 90:6667-6680

Lo EH, Singhal AB, Torchilin VP, Abbott NJ (2001) Drug delivery to damaged brain. Brain Res Rev 38(1-2):140-148

Loeschner K, Hadrup N, Qvortrup K, Larsen A, Gao X, Vogel U, Mortensen A, Lam HR, Larsen EH (2011) Distribution of silver in rats following 28 days of repeated oral exposure to silver nanoparticles or silver acetate. Part Fibre Toxicol $8: 18$

Moghimi SM, Hunter AC (2001) Recognition by macrophages and liver cells of opsonized phospholipid vesicles and phospholipid headgroups. Pharm Res 18:11-18

Moghimi SM, Szebeni J (2003) Stealth liposomes and long circulating nanoparticles: critical issues in pharmacokinetics, opsonization and protein-binding properties. Prog Lipid Res 42:6463-6478

Moghimi SM, Hunter AC, Murray JC (2001) Long-circulating and target-specific nanoparticles: theory to practice. Pharmacol Rev 53:2283-2318

Niidome T, Yamagata M, Okamoto Y, Akiyama Y, Takahashi H, Kawano T, Katayama Y, Niidome Y (2006) PEGmodified gold nanorods with a stealth character for in vivo applications. J Control Rel 114:3343-3347

Oberdorster G, Oberdorster E, Oberdorster J (2005) Nanotoxicology: an emerging discipline evolving from studies of ultrafine particles. Environ Health Perspect 113:7823-7839

Owens DE III, Peppas NA (2006) Opsonization, biodistribution, and pharmacokinetics of polymeric nanoparticles. Int $\mathbf{J}$ Pharm 307:193-202

Pardridge WM (2011) Drug transport in brain via the cerebrospinal fluid. Fluids Barriers CNS 8:17

Pardridge WM (2012) Drug transport across the blood-brain barrier. J Cereb Blood Flow Metab 32(11):1959-1972

Park JH, Lee S, Kim JH, Park K, Kim K, Kwon IC (2008) Polymeric nanomedicine for cancer therapy. Prog Polym Sci 33:1113-1137

Paxinos G, Watson C (2008) The rat brain in stereotaxic coordinates, 6th edn. Elsevier/Academic Press, New York, pp $1-400$
Re F, Gregori M, Masserini M (2012) Nanotechnology for neurodegenerative disorders. Nanomedicine 8(Suppl 1): S51-S58

Roney C, Kulkarni P, Arora V, Antich P, Bonte F, Wu A, Mallikarjuana NN, Manohar S, Liang HF, Kulkarni AR, Sung HW, Sairam M, Aminabhavi TM (2005) Targeted nanoparticles for drug delivery through the blood-brain barrier for Alzheimer's disease. J Control Rel 108(2-3):193-214

Sarin H, Kanevsky AS, Wu H, Brimacombe KR, Fung SH, Sousa AA, Auh S, Wilson CM, Sharma K, Aronova MA, Leapman RD, Griffiths GL, Hall MD (2008) Effective transvascular delivery of nanoparticles across the bloodbrain tumor barrier into malignant glioma cells. J Transl Med 6:80

Schnyder A, Huwyler J (2005) Drug transport to brain with targeted liposomes. NeuroRx. 2:199-207

Templeton NS, Lasic DD, Frederik PM, Strey HH, Roberts DD, Pavlakis GN (1997) Improved DNA: liposome complexes for increased systemic delivery and gene expression. Nat Biotechnol 15:7647-7652

Thurston G, McLean JW, Rizen M, Baluk P, Haskell A, Murphy TJ, Hanahan D, McDonald DM (1998) Cationic liposomes target angiogenic endothelial cells in tumors and chronic inflammation in mice. J Clin Invest 101:71401-71413

Tosi G, Costantino L, Rivasi F, Ruozi B, Leo E, Vergoni AV, Tacchi R, Bertolini A, Vandelli MA, Forni F (2007) Targeting the central nervous system: in vivo experiments with peptide-derivatized nanoparticles loaded with loperamide and rhodamine-123. J Control Rel 122:11-19

Tosi G, Costantino L, Ruozi B, Forni F, Vandelli MA (2008) Polymeric nanoparticles for the drug delivery to the central nervous system. Expert Opin Drug Deliv 5:2155-2174

Vergoni AV, Tosi G, Tacchi R, Vandelli MA, Bertolini A, Costantino L (2009) Nanoparticles as drug delivery agents specific for CNS: in vivo biodistribution. Nanomedicine 5:4369-4377

Zhao W, Zhuang S, Qi XR (2011) Comparative study of the in vitro and in vivo characteristics of cationic and neutral liposomes. Int J Nanomed 6:3087-3098 